\title{
A Gravidade da Doença Afeta os Parâmetros de Repolarização Ventricular em Pacientes com COVID-19
}

\author{
Disease Severity Affects Ventricular Repolarization Parameters in Patients With COVID-19 \\ Mevlut Koc, ${ }^{1 \oplus}$ Hilmi Erdem Sumbul, ${ }^{2}$ Erdinc Gulumsek, ${ }^{2}$ Hasan Koca, ${ }^{1}$ Yurdaer Bulut, ${ }^{3}$ Emre Karakoc, ${ }^{3}$ Tuba \\ Turunc, ${ }^{4}$ Edip Bayrak, ${ }^{4}$ Huseyin Ali Ozturk, ${ }^{2}$ Muhammed Zubeyir Aslan, ${ }^{2}$ Abdullah Orhan Demirtas, ${ }^{1}$ Yahya \\ Kemal Icen ${ }^{1}$ \\ Adana Health Practice and Research Center - Department of Cardiology, ${ }^{1}$ Adana - Turquia \\ Adana Health Practice and Research Center - Department of Internal Medicine, ${ }^{2}$ Adana - Turquia \\ Cukurova University - Faculty of Medicine - Department of Internal Medicine, ${ }^{3}$ Adana - Turquia \\ Adana Health Practice and Research Center - Department of Infectious Disease, ${ }^{4}$ Adana - Turquia
}

\section{Resumo}

Fundamento: Não há estudos avaliando o intervalo $\mathrm{T}_{\text {pico }}-\mathrm{T}_{\text {fim }}$ (Tpe), a relação Tpe/QT e a relação Tpe/QTc para avaliar arritmias cardíacas em pacientes com COVID-19.

Objetivo: Visamos investigar se há alterações nos intervalos QT, QTc e Tpe e nas relações Tpe/QT e Tpe/QTc em pacientes com COVID-19.

Métodos: O estudo incluiu 90 pacientes com infecção por COVID-19 e 30 controles saudáveis pareados por sexo e idade. Foram aferidos os intervalos QT, QTc e Tpe e as relações Tpe/QT e Tpe/QTc. Os participantes incluídos no estudo foram divididos nos seguintes 4 grupos: controles saudáveis (grupo I), pacientes com COVID-19 sem pneumonia (grupo II), pacientes com COVID-19 e pneumonia leve (grupo III) e pacientes com COVID-19 e pneumonia grave (grupo IV). Significância estatística foi definida por valor $p<0,05$.

Resultados: Verificou-se que a frequência cardíaca basal, a presença de hipertensão e diabetes, a contagem de leucócitos, o nitrogênio ureico no sangue, a creatinina, o potássio, o aspartato aminotransferase, a alanina aminotransferase, o NT-proBNP, a proteína $C$ reativa de alta sensibilidade, o dímero-D, a Tncl-as, o intervalo Tpe, a relação Tpe/QT e a relação Tpe/QTc aumentaram do grupo I para o grupo IV e foram significativamente mais altos em todos os pacientes do grupo IV $(p<\mathbf{0 , 0 5})$. A pressão arterial sistólica, a hemoglobina e os níveis de cálcio eram menores no grupo IV e significativamente menores em comparação com os demais grupos $(<0,05)$. Os intervalos QT e QTc eram semelhantes entre grupos. Determinou-se que os níveis elevados de frequência cardíaca, cálcio, dímero-D, NT-proBNP e PCR-as eram significativamente relacionados a Tpe, Tpe/QT e Tpe/QTc.

Conclusões: Em pacientes com COVID-19 e pneumonia grave, o intervalo Tpe, a relação Tpe/QT e a relação Tpe/QTc, que estão entre os parâmetros de repolarização ventricular, foram aumentados, sem prolongação dos intervalos QT e QTc. A partir deste estudo, não podemos definitivamente concluir que as alterações eletrocardiográficas observadas estão diretamente relacionadas à infecção por COVID-19 ou à inflamação, mas sim associadas a cenários graves de COVID-19, que podem envolver outras causas de inflamação e comorbidades. (Arq Bras Cardiol. 2020; 115(5):907-913)

Palavras-chave: COVID-19/complicações; Betacoronavírus; Doenças Cardiovasculares; Diabetes Melitus; Hipertensão; Pneumonia; Estudo Comparativo.

\footnotetext{
Abstract

Background: There is no study evaluating the $T_{\text {peak }} T_{\text {end }}$ (Tpe) interval, Tpe/QT ratio, and Tpe/QTC ratio to assess cardiac arrhythmias in patients with COVID-19.
}

Objective: We aimed to examine whether there is a change in QT, QTC, Tpe interval, Tpe/QT ratio, and Tpe/QTC ratio in patients with COVID-19. Methods: The study included 90 patients with COVID-19 infection and 30 age-and-sex-matched healthy controls. QT, QTC, Tpe interval, Tpe/

Correspondência: Mevlut Koc •

University of Health Sciences - Adana Health Practice and Research Center - Department of Cardiology - Adana City Hospital Deparment of cardio Adana Adana 01100 - Turquia

E-mail: drmevlutkoc@gmail.com

Artigo recebido em 19/05/2020, revisado em 21/07/2020, aceito em 05/08/2020

DOI: https://doi.org/10.36660/abc.20200482 
QT ratio, and Tpe/QTc ratio were measured. The participants included in the study were divided into the following 4 groups: healthy controls (group I), patients with COVID-19 without pneumonia (group II), patients with COVID-19 and mild pneumonia (group III), and patients with COVID-19 and severe pneumonia (group IV). Statistical significance was set at $p<0.05$

Results: It was found that baseline heart rate, presence of hypertension and diabetes, white blood cell count, blood urea nitrogen, creatinine, potassium, aspartate aminotransferase, alanine aminotransferase, NT-proBNP, high sensitive C reactive protein, D-dimer, hs-cTnl, Tpe, Tpe/QT, and Tpe/QTC increased from group I to group IV, and they were significantly higher in all patients in group IV ( $p<0.05$ ). Systolic-diastolic blood pressure, hemoglobin, and calcium levels were found to be lowest in group IV and significantly lower than in other groups $(<0.05)$. QT and QTC intervals were similar between groups. It was determined that increased heart rate, calcium, D-dimer, NT-proBNP and hs-CRP levels were significantly related to Tpe, Tpe/QT, and Tpe/QTC.

Conclusions: In patients with COVID-19 and severe pneumonia, Tpe, Tpe/QT ratio, and Tpe/QTC ratio, which are among ventricular repolarization parameters, were found to be increased, without prolonged QT and QTc intervals. In this study, we cannot definitively conclude that the ECG changes observed are directly related to COVID-19 infection or inflammation, but rather associated with severe COVID-19 scenarios, which might involve other causes of inflammation and comorbidities. (Arq Bras Cardiol. 2020; 115(5):907-913)

Keywords: COVID-19/complications; Betacoronavirus, Cardiovascular Diseases; Diabetes Mellitus; Hypertension; Pneumonia; Comparative Study.

Full texts in English - http://www.arquivosonline.com.br

\section{Introdução}

Durante os últimos meses de 2019, surgiu uma nova pandemia causada pela síndrome respiratória aguda grave coronavírus 2 (SARS-CoV-2), e seus efeitos ainda estão em andamento. Essa doença, denominada doença coronavírus 2019 (COVID-19), afeta principalmente o trato respiratório, mas tem uma taxa significativa (12\% a $28 \%$ ) de envolvimento cardíaco. ${ }^{1-4}$ Níveis aumentados de troponina T cardíaca (TnTc), troponina cardíaca I (Tncl), e de Tncl e TnTc de alta sensibilidade (Tncl-as e TnTc-as) ${ }^{1,2,4}$ e NT-proBNP ${ }^{5}$ foram encontrados em pacientes com envolvimento cardíaco. A mortalidade aumenta nos pacientes com envolvimento cardíaco. ${ }^{1,6-8} \mathrm{O}$ envolvimento cardíaco é multifatorial em pacientes com COVID-19. 1,4,9-16 Uma vez que o envolvimento cardíaco está associado à mortalidade, é possível predizer um aumento da mortalidade devido à arritmia nestes pacientes. De fato, os pacientes com COVID-19 apresentaram arritmias fatais. ${ }^{1-3,9}$ Apesar disso, nenhum parâmetro ou classificação clara foi relatada para fornecer informações sobre a frequência das arritmias ou para predizê-las nesses pacientes. Só foi recomendado medir o QT e o QT corrigido (QTc) com antecedência, a fim de reduzir eventos arrítmicos fatais antes de iniciar a hidroxicloroquina e a azitromicina que têm sido usadas na profilaxia e tratamento de COVID-19. ${ }^{17}$

A repolarização ventricular prolongada ou comprometida está associada a arritmias potencialmente fatais, como a taquicardia ventricular (TV) e a fibrilação ventricular (FV). Existem muitos parâmetros de eletrocardiograma (ECG) relacionados à despolarização e à repolarização ventricular. Os parâmetros utilizados na prática clínica são os intervalos QT e QTc, a dispersão de QT e de QTc e o intervalo $T_{\text {pico }}-T_{\text {fim }}$ (Tpe). As relações Tpe/QT e Tpe/QTc obtidas a partir destes parâmetros estão associadas à dispersão transmural ventricular durante a repolarização. ${ }^{18} \mathrm{O}$ intervalo Tpe elevado indica disseminação anormal na repolarização ventricular e está associado ao risco aumentado de arritmia ventricular. ${ }^{19}$ Até onde sabemos, não há estudos sobre o QT, o QTc, o intervalo Tpe, a relação Tpe/QT e a relação Tpe/QTc com respeito ao efeito do COVID-19 nos parâmetros de repolarização ventricular. Portanto, o objetivo do nosso estudo foi o de investigar se existem alterações no
QT, no QTc, no intervalo Tpe, na relação Tpe/QT e na relação Tpe/QTc em pacientes com COVID-19.

\section{Materiais e Métodos}

Foram examinados retrospectivamente 120 pacientes com diagnóstico de COVID-19, internados em terapia intensiva, serviço de internação ou clínicas de pandemia COVID-19, entre 15 de março e 20 de abril de 2020 e submetidos ao ECG de admissão. Após a aplicação dos critérios de exclusão, o estudo incluiu 30 pacientes com COVID-19 e pneumonia grave (grupo IV, 20 homens e 10 mulheres, idade média 61,2 $\pm 10,1$ anos), 30 pacientes com COVID-19 e pneumonia leve (grupo III, 18 homens e 12 mulheres, idade média 64,8 $\pm 12,3$ anos), 30 pacientes com COVID-19 sem pneumonia (grupo II, 19 homens e 11 mulheres, idade média 65,2 \pm 14,2 anos) e 30 controles saudáveis (17 homens e 13 mulheres, idade média $63,5 \pm 13,5$ anos). Para os pacientes com COVID-19 examinados neste estudo, foram considerados como critérios de exclusão os seguintes fatores: grupo de idade pediátrica (< 18 anos); ausência de medição de Tpe e QTc; doença arterial coronariana ou síndrome coronariana aguda conhecidas; doença cardíaca valvar leve a avançada; insuficiência cardíaca sistólica; qualquer tratamento médico conhecido por prolongar ou encurtar os intervalos QT ou QTC e histórico pessoal ou familiar de síncope ou parada súbita cardíaca. O estudo foi realizado de acordo com a Declaração de Helsinque e foi aprovado pelo comitê de ética local.

Os parâmetros demográficos, clínicos e bioquímicos e o ECG de 12 derivações de todos os pacientes foram obtidos de seus prontuários. Os dados demográficos de todos os pacientes como sexo, frequência cardíaca (FC) basal, pressão arterial sistólica (PAS) e pressão arterial diastólica (PAD), foram obtidos dos arquivos. Usando parâmetros bioquímicos de rotina, contagem de leucócitos, hemograma, nível sanguíneo de glicose, testes de função renal, aspartato aminotransferase (AST), alanina aminotransferase (ALT), nível sérico de cálcio, colesterol de lipoproteína de baixa densidade (LDL), proteína $C$ reativa de alta sensibilidade (PCR-as), dímero- $D, N$-terminal do peptídeo natriurético cerebral tipo B (NT-pro-BNP) e Tnclas foram registrados. 


\section{Avaliações de Eletrocardiograma de 12 Derivações}

ECG de 12 derivações, realizados usando uma máquina de ECG MAC 2000 (GE medical systems information technologies, Inc., WI, EUA), em ritmo sinusal, com velocidade de $25 \mathrm{~mm} / \mathrm{seg}$ e calibração padrão de $1 \mathrm{mv} / 10 \mathrm{~mm}$, foram obtidos dos arquivos para todos os indivíduos. Para o intervalo QT, foi calculado o tempo do começo da onda QRS até o ponto onde a onda T se fundia com a linha isoelétrica. QTC foi calculado utilizando-se a fórmula de Bazett $\left(Q T_{C}=Q T_{C} /\right.$ $\sqrt{ } R-R)$. O limite superior do normal para QTc foi aceito como 450 e 460 ms para homens e mulheres, respectivamente. ${ }^{20}$ O intervalo Tpe foi definido como o tempo do pico da onda T até o ponto onde a onda $\mathrm{T}$ se junta e termina com a linha isoelétrica. As medições foram feitas principalmente a partir de V5. Nos casos em que V5 não era adequado para medição (amplitude $<1,5 \mathrm{~mm}$ ), as medições foram feitas de V4 ou V6. ${ }^{21}$ As relações Tpe/QT e Tpe/QTC foram calculadas de acordo com essas medições. Todos os exames de ECG em ritmo sinusal foram avaliados por dois cardiologistas com pelo menos 5 anos de experiência em eletrofisiologia, que avaliam $\geq 2.000$ pacientes com arritmias anualmente e que não conheciam o paciente ou a clínica.

\section{Análise Estatística}

O teste de Shapiro-Wilk foi usado para distribuição normal das variáveis contínuas. As variáveis contínuas dos dados dos grupos foram indicadas como média \pm desvio padrão ou mediana e intervalo interquartil. As variáveis categóricas foram especificadas como números e porcentagens. As variáveis contínuas que apresentavam distribuição normal foram comparadas pelo teste ANOVA unidirecional, enquanto o teste de Kruskal-Wallis foi usado para comparar amostras sem distribuição normal. Para os dados com distribuição normal, os testes de Scheffe e Games-Howell foram usados para comparações múltiplas de grupos, em relação à homogeneidade das variâncias. Para dados não normalmente distribuídos, o teste U Mann-Whitney ajustado de Bonferroni foi usado para comparações múltiplas entre grupos. Foi usado o teste de qui-quadrado para comparar as variáveis categóricas. As análises de correlação de Pearson e Spearman foram realizadas para determinar os parâmetros relacionados ao intervalo Tpe e as relações de Tpe/QT e Tpe/QTc. Foi realizada a análise de regressão linear para os parâmetros que estavam mais relacionados ao intervalo Tpe e às relações Tpe/QT e Tpe/QTc na análise univariada. Para evitar problemas de multicolinearidade, cada parâmetro de repolarização ventricular foi analisado separadamente em modelos diferentes. Todos os modelos foram ajustados por sexo, idade e fatores de risco cardiovascular. O coeficiente kappa foi utilizado para avaliar a variabilidade interobservador e intraobservador de todas as medidas eletrocardiográficas.

\section{Resultados}

Conforme previamente descrito, os dados do estudo foram divididos em 4 grupos e comparados. As medidas eletrocardiográficas foram obtidas com sucesso em todos os pacientes incluídos no estudo. Os valores do kappa de Cohen que avaliam a variabilidade interobservador e intraobservador foram superiores a 90\% para todos os critérios do ECG.

\section{Dados Demográficos e Clínicos dos Grupos do Estudo}

Quando os dados demográficos foram comparados de acordo com os grupos do estudo, a distribuição de idade e sexo foi semelhante entre os grupos. A frequência de hipertensão e diabetes mellitus era mais alta no grupo IV. Entre os parâmetros clínicos, foi demonstrado que os valores de PAS e PAD foram os mais baixos nos pacientes do grupo IV e eram significativamente menores em comparação com os demais grupos (Tabela 1). Também foi demonstrado que o valor basal da FC aumentou do grupo I para o grupo IV e os valores foram significativamente maiores nos pacientes do grupo IV em comparação com os demais grupos (Tabela 1). A PAS, a PAD, e os valores basais de FC dos grupos I, II, e III eram semelhantes (Tabela 1).

\section{Dados Laboratoriais dos Grupos do Estudo}

Os parâmetros laboratoriais, como a contagem de leucócitos, o nitrogênio ureico no sangue, creatinina, potássio, AST, ALT, PCR-as, dímero-D, NT-proBNP e Tncl-as aumentaram do grupo I para o grupo IV e foram significativamente mais altos nos pacientes do grupo IV em comparação com os demais grupos (Tabela 1). Além disso, a contagem de leucócitos, os níveis de AST, ALT e dímero-D foram significativamente maiores em comparação com o grupo I e o grupo II. Foi determinado que os níveis de hemoglobina e de cálcio diminuíram do grupo I para o grupo IV e foram significativamente menores nos pacientes do grupo IV em comparação com os demais grupos. Também foram menores no grupo III do que nos grupos I e II (Tabela 1).

\section{Dados Eletrocardiográficos dos Grupos do Estudo}

Quando os dados de ECG foram comparados de acordo com os grupos do estudo, os intervalos QT e QTc foram semelhantes em todos os grupos (Tabela 2). Apenas 1 paciente apresentou QTc > 500 ms e 1 paciente apresentou QTc > $460 \mathrm{~ms}$. Os valores de QTc de todos os outros pacientes eram normais. O intervalo Tpe e as relações Tpe/QT e Tpe/QTc aumentaram do grupo I para o grupo IV e foram significativamente maiores em todos os pacientes do grupo IV em comparação com os demais grupos (Tabela 2).

\section{A Determinação dos Parâmetros Relacionados ao Intervalo Tpe, à Relação Tpe/QT e à Relação Tpe/QTc}

Foi realizada análise de correlação para determinar os parâmetros relacionados ao intervalo Tpe, à relação Tpe/ QT e à relação Tpe/QTc. A Tabela 3 resume os parâmetros relacionados ao intervalo $\mathrm{Tpe}$, à relação $\mathrm{Tpe} / \mathrm{QT}$ e à relação Tpe/QTc na análise de correlação. Foi realizada a análise de regressão linear para determinar os parâmetros significativamente relacionados ao intervalo Tpe, à relação Tpe/QT e à relação Tpe/QTc na análise de correlação (Tabela 4). Como resultado desta análise, foi verificado que os níveis basais de FC, dímero-D e Tncl-as eram positiva e significativamente associados ao intervalo Tpe, à relação Tpe/ QT e à relação Tpe/QTc. O nível sérico de cálcio foi negativa e significativamente correlacionado com o intervalo Tpe e com a relação Tpe/QTc. Concomitantemente, o NT-proBNP e a relação Tpe/QTc foram positiva e significativamente 


\begin{tabular}{|c|c|c|c|c|c|}
\hline Variável & $\begin{array}{c}\text { Grupo I } \\
n=30\end{array}$ & $\begin{array}{c}\text { Grupo II } \\
n=30\end{array}$ & $\begin{array}{c}\text { Grupo III } \\
n=30\end{array}$ & 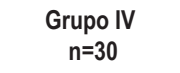 & $\mathrm{p}$ \\
\hline Idade (anos) & $63,5 \pm 13,5$ & $65,2 \pm 14,2$ & $64,8 \pm 12,3$ & $61,2 \pm 10,1$ & 0,627 \\
\hline Sexo (masculino/feminino) & $17 / 13$ & 19/11 & $18 / 12$ & $20 / 10$ & 0,506 \\
\hline Hipertensão, n (\%) & $0(0 \%)$ & $7(23 \%)$ & $15(50 \%)$ & $17(57 \%)$ & $<0,001$ \\
\hline Diabetes mellitus, n (\%) & $0(0 \%)$ & $6(20 \%)$ & $7(23 \%)$ & $11(38 \%)$ & $<0,001$ \\
\hline Tabagismo atual, $\mathrm{n}(\%)$ & $0(0 \%)$ & $14(47 \%)$ & $15(50 \%)$ & $12(40 \%)$ & 0,425 \\
\hline PAS (mmHg) & $125 \pm 11^{a}$ & $130 \pm 10,1^{\beta}$ & $136 \pm 14^{*}$ & $108 \pm 30$ & $<0,001$ \\
\hline $\mathrm{PAD}(\mathrm{mmHg})$ & $76,9 \pm 4,8^{a}$ & $79,9 \pm 7,6^{\beta}$ & $80,2 \pm 7,5^{*}$ & $62,3 \pm 23,1$ & $<0,001$ \\
\hline Pulso (bpm) & $67 \pm 8,2^{a}$ & $68 \pm 9,1^{\beta}$ & $75,6 \pm 12,1^{*}$ & $89,6 \pm 19,5$ & $<0,001$ \\
\hline Contagem de leucócitos (UL) & $9039 \pm 1188^{\mathrm{a}, \mathrm{*}}$ & $1097 \pm 1516^{\beta, \triangle}$ & $1277 \pm 1484^{*}$ & $1906 \pm 2698$ & $<0,001$ \\
\hline Hemoglobina (gr/dL) & $13,3 \pm 1,05^{a, *}$ & $13,4 \pm 1,58^{\beta, \Delta}$ & $12,7 \pm 0,81^{*}$ & $10,6 \pm 0,74$ & $<0,001$ \\
\hline Glicose (mg/dL) & $105 \pm 13$ & $138 \pm 13$ & $141 \pm 11$ & $138 \pm 12$ & 0,172 \\
\hline Nitrogênio ureico no sangue (mg/dL) & $25,3 \pm 6,7^{a}$ & $28,1 \pm 8,8^{\beta}$ & $37,2 \pm 25^{*}$ & $66,9 \pm 80$ & 0,001 \\
\hline Creatinina $(\mathrm{mg} / \mathrm{dL})$ & $0,60 \pm 0,07^{a}$ & $0,62 \pm 0,07^{\beta}$ & $0,67 \pm 0,18^{*}$ & $1,08 \pm 0,80$ & $<0,001$ \\
\hline Sódio (mEq/L) & $140 \pm 4,0$ & $140 \pm 3,7$ & $140 \pm 6,8$ & $140 \pm 2,5$ & 0,986 \\
\hline Potássio (mEq/L) & $4,31 \pm 0,33^{a}$ & $4,34 \pm 0,34^{\beta}$ & $4,30 \pm 0,68^{*}$ & $4,74 \pm 0,58$ & 0,002 \\
\hline Cálcio (mg/dL) & $9,45 \pm 0,50^{a, *}$ & $9,47 \pm 0,56^{\beta, \Delta}$ & $8,38 \pm 0,82^{*}$ & $7,99 \pm 1,20$ & $<0,001$ \\
\hline AST (u/L) & $28(26-29)^{a, *}$ & $28(28-29)^{\beta, \Delta}$ & $47(43-49)^{*}$ & $50(44-56)$ & $<0,001$ \\
\hline $\mathrm{ALT}(\mathrm{u} / \mathrm{L})$ & $27(23-27)^{a, ;}$ & $26(23-27)^{\beta, \Delta}$ & $34(33-27)^{*}$ & $37(36-40)$ & $<0,001$ \\
\hline Colesterol LDL (mg/dL) & $117 \pm 25$ & $115 \pm 25$ & $117 \pm 24$ & $113 \pm 25$ & 0,911 \\
\hline PCR-as (mg/dL) & $1,2(1,0-1,4)^{a}$ & $1,2(1,0-1,4)^{\beta}$ & $2,1(1,5-3,1)^{*}$ & $17(11-22)$ & $<0,001$ \\
\hline Dímero-D (ng/mL) & $4(3-36)^{a, *}$ & $4(4-35)^{\beta, \Delta}$ & $499(34-725)^{*}$ & $750(499-1550)$ & $<0,001$ \\
\hline NT-proBNP (pg/mL) & $23(10-33)^{a}$ & $21(11-34)^{\beta}$ & $100(41-111)^{*}$ & $123(110-567)$ & 0,033 \\
\hline Tncl-as (ng/L) & $5(3-13)^{a}$ & $5(3-14)^{\beta}$ & $16(14-30)^{*}$ & $20(14-156)$ & 0,005 \\
\hline
\end{tabular}

Os valores são apresentados como média \pm desvio padrão, mediana e intervalo interquartil ou n (\%). ALT: alanina aminotransferase; AST: aspartato aminotransferase; LDL: lipoproteína de baixa densidade; NT-proBNP: N-terminal do peptídeo natriurético cerebral tipo B; PAD: pressão arterial diastólica; PAS: pressão arterial sistólica, PCR-as: proteina C reativa de alta sensibilidade; Tncl-as: troponina cardíaca I de alta sensibilidade. Grupo I: controles saudáveis, grupo Il: pacientes com COVID-19 sem pneumonia, grupo III: pacientes com COVID-19 e pneumonia leve, grupo IV: pacientes com COVID-19 e pneumonia grave. $\alpha=$ diferença significativa entre grupo Ie grupo IV $(p<0,05)$. $¥$ = diferença significativa entre grupo I e grupo III $(p<0,05)$. $\$$ diferença significativa entre grupo I e grupo II $(p<0,05)$. $\beta=$ diferença significativa entre grupo II e grupo IV $(p<0,05) . \Delta=$ diferença significativa entre grupo II e grupo III $(p<0,05)$. $*$ * diferença significativa entre grupo III e grupo IV $(p<0,05)$.

relacionados. Estatisticamente, a relação mais significativa foi encontrada entre a relação Tpe/QTc e o dímero-D (Tabela 4).

\section{Discussão}

O achado principal do nosso estudo é que, em pacientes com COVID-19 e pneumonia grave, o intervalo Tpe e as relações Tpe/QT e Tpe/QTc são elevados, sem prolongação dos intervalos QT e QTc. Até onde sabemos, este é o primeiro estudo na literatura a demonstrar aumento do intervalo Tpe e das relações Tpe/QT e Tpe/QTc, que estão entre os parâmetros de repolarização ventricular, em pacientes com COVID-19.
A infecção por COVID-19 envolve principalmente as vias aéreas, mas complicações cardiovasculares significativas também podem ocorrer. ${ }^{1-3,9,10}$ Não seria correto explicar o envolvimento cardíaco ou as complicações que ocorrem nesta doença como um mecanismo único; a lesão cardíaca é considerada multifatorial. ${ }^{4}$ Os possíveis mecanismos para o envolvimento cardíaco podem ser resumidos da maneira seguinte: i) miocardite viral direta como o mecanismo mais comumente considerado; ;,9-11 ii) hipotensão e FC elevada; ${ }^{3}$ iii) hipóxia; ${ }^{14}$ iv) inflamação elevada e liberação de citocina; ${ }^{14}$ v) regulação negativa dos receptores ECA- $2 ;^{13}$ vi) toxicidade de medicamentos (cloroquina, hidroxicloroquina, 


\begin{tabular}{|c|c|c|c|c|c|}
\hline Variável & $\begin{array}{c}\text { Grupo I } \\
n=30\end{array}$ & $\begin{array}{c}\text { Grupo II } \\
n=30\end{array}$ & $\begin{array}{c}\text { Grupo III } \\
n=30\end{array}$ & 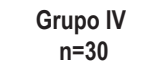 & $\mathrm{p}$ \\
\hline Intervalo QT, tempo (ms) & $367 \pm 49$ & $380 \pm 21$ & $381 \pm 24$ & $382 \pm 51$ & 0,338 \\
\hline Intervalo QTc, tempo (ms) & $405 \pm 23$ & $406 \pm 34 \beta, \Delta$ & $406 \pm 15$ & $407 \pm 16$ & 0,989 \\
\hline Intervalo Tpe, tempo (ms) & $70,3 \pm 7,1 \mathrm{a}$ & $72,7 \pm 7,7 \beta$ & $74,1 \pm 8,5^{*}$ & $90,1 \pm 9,2$ & $<0,001$ \\
\hline Relação Tpe/QT & $0,186 \pm 0,021 a$ & $0,191 \pm 0,023 \beta$ & $0,203 \pm 0,051^{*}$ & $0,235 \pm 0,034$ & $<0,001$ \\
\hline Relação Tpe/QTc & $0,188 \pm 0,022 a$ & $0,186 \pm 0,024 \beta$ & $0,200 \pm 0,018^{*}$ & $0,216 \pm 0,029$ & $<0,001$ \\
\hline
\end{tabular}

Os valores são apresentados como média \pm desvio padrão ou n (\%)

Grupo I: controles saudáveis, grupo II: pacientes com COVID-19 sem pneumonia, grupo III: pacientes com COVID-19 e pneumonia leve, grupo IV: pacientes com COVID-19 e pneumonia grave. $\alpha=$ diferença significativa entre grupo I e grupo IV $(p<0,05)$. $¥=$ diferença significativa entre grupo I e grupo III $(p<0,05)$. $\nabla=$ diferença significativa entre grupo I e grupo II $p<0.05)$. $\beta=$ diferença significativa entre grupo II e grupo IV $(p<0,05)$. $\Delta=$ diferença significativa entre grupo II e grupo III $(p<0,05) . *{ }^{*}=$ diferença significativa entre grupo III e grupo IV $\left.p<0.05\right)$.

Tabela 3 - Análises de correlação para parâmetros associados a intervalo Tpe, relação Tpe/QT e relação Tpe/QTc

\begin{tabular}{lccccccc} 
& \multicolumn{2}{c}{ Intervalo Tpe } & & Tpe/QT & & Tpe/QTc \\
\cline { 2 - 8 } & $\mathbf{r}$ & $\mathbf{p}$ & $\mathbf{r}$ & $\mathbf{p}$ & $\mathbf{r}$ & $\mathbf{p}$ \\
\hline Pressão arterial sistólica $(\mathrm{mmHg})$ & $-0,296$ & 0,001 & $-0,175$ & 0,056 & $-0,149$ & 0,105 \\
\hline Pressão arterial diastólica $(\mathrm{mmHg})$ & $-0,218$ & 0,017 & $-0,187$ & 0,040 & $-0,183$ & 0,046 \\
\hline Pulso (bpm) & 0,298 & 0,001 & 0,309 & 0,001 & 0,125 & 0,424 \\
\hline Creatinina (mg/dL) & 0,279 & 0,002 & 0,223 & 0,014 & 0,247 & 0,007 \\
\hline Potássio (mEq/L) & 0,274 & 0,002 & 0,299 & 0,001 & 0,120 & 0,405 \\
\hline Cálcio (mg/dL) & $-0,461$ & $<0,001$ & $-0,241$ & 0,008 & $-0,287$ & 0,002 \\
\hline PCR-as (mg/dL) & 0,245 & 0,007 & 0,208 & 0,023 & 0,219 & 0,012 \\
\hline Dímero-D (ng/mL) & 0,431 & $<0,001$ & 0,298 & 0,001 & 0,569 & $<0,001$ \\
\hline NT-proBNP (pg/mL) & 0,192 & 0,035 & 0,190 & 0,045 & 0,351 & $<0,001$ \\
\hline Tncl-as (ng/L) & 0,185 & 0,042 & 0,210 & 0,019 & 0,255 & 0,005 \\
\hline
\end{tabular}

NT-proBNP: N-terminal do peptídeo natriurético cerebral tipo B; PCR-as: proteina C reativa de alta sensibilidade; Tncl-as: troponina cardíaca I de alta sensibilidade.

eritromicina, etc. $)^{15-16}$ e vii) aumento da liberação endógena de catecolaminas. ${ }^{12}$ Embora todos estes parâmetros não tenham sido observados no nosso estudo, foram verificados aumentos dos níveis de Tncl-as e NT-proBNP, que sugere envolvimento miocárdico; aumento na contagem de leucócitos e PCR-as, evidenciando o processo de inflamação; aumento da FC com diminuição da PAS e da PAD, evidenciando o estado hemodinâmico, evoluindo do grupo controle para o grupo com pneumonia grave, em consonância com a literatura. Além disso, no nosso estudo, foi verificado um aumento no nível do dímero-D em pacientes com maior gravidade da doença.

A mortalidade é paralela ao aumento do envolvimento cardíaco em pacientes com COVID-19..$^{1,6-8}$ Assim como nas doenças cardiovasculares, a causa mais comum de mortalidade cardíaca em pacientes com COVID-19 são os eventos arrítmicos. Em muitos estudos, foi relatado que os pacientes com COVID-19 e envolvimento cardíaco têm diferentes frequências e tipos de arritmias cardíacas. ${ }^{1-3,9}$
Ainda não existe um mecanismo e uma classificação clara das arritmias para essa doença. Num estudo com 187 pacientes, Guo et al., ${ }^{2}$ relataram uma taxa de envolvimento cardíaco de 27,8\%, TV ou FV sendo presente em 5,9\% destes pacientes. Zhou et al., ${ }^{3}$ relataram que a taxa de envolvimento cardíaco foi de 17\% em 191 pacientes, e 1\% desses pacientes apresentou FC > $125 \mathrm{bpm}$. Shi et al., ${ }^{1}$ relataram que a taxa de envolvimento cardíaco foi de 19,7\% em 416 pacientes, a depressão do segmento ST sendo encontrada em 0,7\% desses pacientes. Wang et al., ${ }^{9}$ relataram uma frequência de $16,7 \%$ de eventos arrítmicos, em 118 pacientes.

O mecanismo mais importante da fisiopatologia da arritmia ventricular em pacientes com infecção por COVID-19 é semelhante ao das arritmias em pacientes com miocardite aguda. ${ }^{1,9-11}$ Assim como na miocardite aguda, os motivos mais importantes das arritmias são o aumento da Tncl-as e a diminuição da função ventricular esquerda, devido ao aumento do dano miocárdico durante o período agudo, bem como 


\begin{tabular}{|c|c|c|c|c|c|c|}
\hline & \multicolumn{2}{|c|}{ Intervalo Tpe } & \multicolumn{2}{|c|}{ Tpe/QT } & \multicolumn{2}{|c|}{ Tpe/QTc } \\
\hline & $\beta$ & $\mathrm{p}$ & $\beta$ & $\mathrm{p}$ & $\beta$ & $\mathrm{p}$ \\
\hline Pressão arterial sistólica (mmHg) & $-0,092$ & 0,236 & $-0,056$ & 0,530 & $-0,013$ & 0,865 \\
\hline Pressão arterial diastólica (mmHg) & $-0,017$ & 0,827 & $-0,057$ & 0,536 & $-0,698$ & 0,487 \\
\hline Pulso (bpm) & 0,271 & 0,001 & 0,286 & 0,001 & 0,205 & 0,007 \\
\hline Creatinina (mg/dL) & 0,143 & 0,054 & 0,153 & 0,074 & 0,054 & 0,499 \\
\hline Potássio (mEq/L) & 0,105 & 0,168 & 0,175 & 0,158 & 0,158 & 0,168 \\
\hline Cálcio (mg/dL) & $-0,298$ & $<0,001$ & $-0,103$ & 0,263 & $-0,241$ & 0,002 \\
\hline PCR-as (mg/dL) & 0,078 & 0,306 & 0,113 & 0,188 & 0,021 & 0,773 \\
\hline Dímero-D (ng/mL) & 0,342 & $<0,001$ & 0,271 & 0,002 & 0,493 & $<0,001$ \\
\hline NT-proBNP (pg/mL) & 0,114 & 0,125 & 0,055 & 0,526 & 0,233 & 0,001 \\
\hline Tncl-as (ng/L) & 0,235 & 0,002 & 0,205 & 0,010 & 0,198 & 0,012 \\
\hline
\end{tabular}

NT-proBNP: N-terminal do peptídeo natriurético cerebral tipo B; PCR-as: proteína $C$ reativa de alta sensibilidade; Tncl-as: troponina cardiaca I de alta sensibilidade. $R_{\text {aiustado }}^{2}$ para intervalo Tpe, Tpe/QT e Tpe/QTc como 0,426, 0,446 e 0,487, respectivamente.

a fibrose atrial e ventricular ocorrendo no período tardio. ${ }^{22}$ Em estudos realizados em pacientes com miocardite aguda em anos prévios, foram verificados aumentos dos intervalos QT, QTc e Tpe e as relações Tpe/QT e Tpe/QTc durante o período agudo. ${ }^{23,24}$ Até onde sabemos, não existem estudos que pesquisem QT, QTc, o intervalo Tpe, a relação Tpe/QT e a relação Tpe/QTc em casos de miocardite ou envolvimento cardíaco em pacientes com COVID-19. Em nosso estudo, os níveis de Tncl-as eram significativamente mais altos nos pacientes com COVID-19 e pneumonia grave. No nosso estudo, não foram realizadas análise e classificação das arritmias porém, os parâmetros de repolarização ventricular dos pacientes, que podem predizer eventos arrítmicos com antecedência, foram avaliados na admissão. Foi verificado que o intervalo Tpe, a relação Tpe/QT e a relação Tpe/QTc, que estão entre os parâmetros de repolarização ventricular, aumentaram com a atividade e gravidade da doença, sendo bem maiores em pacientes com pneumonia grave. Além disso, verificou-se que houve uma associação positiva e significativa entre Tncl-as e o intervalo Tpe, a relação Tpe/QT e a relação Tpe/QTc, o que corrobora estudos que mostram que a frequência de arritmias aumentou em pacientes com TnTc-as elevada.

Há muitos parâmetros relacionados à atividade da doença e ao seu prognóstico em pacientes com COVID-19. A maioria dos parâmetros associados à atividade e ao prognóstico também estão associados com o envolvimento cardíaco. No nosso estudo, a atividade da doença foi associada à presença e gravidade da pneumonia. Além disso, os parâmetros comprometidos de repolarização ventricular foram positiva e significativamente relacionados ao aumento de $\mathrm{FC}^{3}$ NT-proBNP, ${ }^{8}$ dímero-D ${ }^{5}$ e Tncl-as, ${ }^{1-5}$ que são intimamente relacionados à atividade da COVID-19 na literatura. Portanto, hipotetizamos que o prolongamento elevado da repolarização miocárdica nos pacientes com COVID-19 pode ser afetado pela atividade da doença e que os eventos arrítmicos nesses pacientes pudessem ser previstos com antecedência.

\section{Limitações}

Há algumas limitações importantes no nosso estudo, incluindo o desenho retrospectivo do estudo e o número de pacientes incluídos. Além disso, os eventos arrítmicos e os parâmetros de acompanhamento clínico não foram avaliados, devido ao número pequeno de pacientes e à falta de acompanhamento clínico. Estudos prospectivos com mais pacientes podem fornecer informações mais significativas. No nosso estudo, os medicamentos e os tratamentos médicos que prolongam o QT foram considerados como critérios de exclusão, mas não foi realizada nenhuma avaliação genética para QT longo ou curto. Esta canalopatia hereditária pode não ser muito significativa, devido à sua raridade. Não foi realizada ressonância magnética para o envolvimento cardíaco ou a miocardite devido à COVID-19. Outra limitação importante do nosso estudo foi a impossibilidade de avaliar os efeitos de drogas, como a hidroxicloroquina e a azitromicina, que são frequentemente utilizadas para tratar COVID-19, na repolarização ventricular.

\section{Conclusão}

Embora o problema principal relacionado à mortalidade e à morbidade em pacientes com COVID-19 seja a doença pulmonar aguda, as evidências disponíveis indicam que um em cada cinco pacientes com COVID-19 apresenta dano miocárdico. O nosso estudo demonstrou que, em adição aos estudos prévios sobre COVID-19 na literatura, o distúrbio da repolarização miocárdica ocorreu em adição ao aumento do dano miocárdico em pacientes com pneumonia grave. Em pacientes com COVID-19, foi verificado que o intervalo Tpe, a relação Tpe/QT e a relação Tpe/QTc, que estão entre os parâmetros de dispersão transmural de repolarização ventricular, eram elevados, sem prolongação dos intervalos QT e QTc. Isso foi mais pronunciado em pacientes com COVID-19 grave e pneumonia grave e pode estar associado ao aumento 
da inflamação e ao dano miocárdico. Para pacientes com COVID-19, principalmente aqueles com pneumonia grave, deve-se ter em mente que pode ocorrer prolongamento na repolarização ventricular. Neste estudo, não podemos definitivamente concluir que as alterações eletrocardiográficas observadas estão diretamente relacionadas à infecção por COVID-19 ou à inflamação, mas sim associadas a cenários graves de COVID-19, que podem envolver outras causas de inflamação e comorbidades.

\section{Contribuição dos Autores}

Concepção e desenho da pesquisa: Sumbul HE, Koca H; Obtenção de dados: Gulumsek E, Koca H, Turunc T, Bayrak E, Ozturk HA, Demirtas AO; Análise e interpretação dos dados: Koc M, Bulut Y, Bayrak E, Aslan MZ; Análise estatística: Koc

\section{Referências}

1. Shi S, Qin M, Shen B, Cai Y, Liu T, Yang F, et al. Association of cardiac injury with mortality in hospitalized patients with COVID-19 in Wuhan, China. JAMA. 2020; 5(7):802-10.

2. Guo T, Fan Y, Chen M, Wu X, Zhang L, He T, etal. Cardiovascular implications of fatal outcomes of patients with coronavirus disease 2019 (COVID-19). JAMA Cardiol. 2020; 5(7):811-8

3. Zhou F, Yu T, Du R, Fan G, Liu Y, Liu Z, et al. Clinical course and risk factors for mortality of adult inpatients with COVID-19 in Wuhan, China: a retrospective cohort study. Lancet. 2020; 395(10229):1054-62.

4. Kochi AN, Tagliari AP, Forleo GB, Fassini GM, Tondo C. Cardiac and arrhythmic complications in Covid-19 patients. J Cardiovasc Electrophysiol. 2020; 31(5):1003-8.

5. Lippi G, Lavie CJ, Sanchis-Gomar F. Cardiac troponin I in patients with coronavirus disease 2019 (COVID-19): Evidence from a meta-analysis. Prog Cardiovasc Dis. 2020; 63(3):390-1.

6. Du RH, Liang LR, Yang CQ, Wang W, Cao TZ, Li M, et al. Predictors of Mortality for Patients with COVID-19 Pneumonia Caused by SARS-CoV-2: A Prospective Cohort Study. Eur Respir J. 2020; 55(5):2000524.

7. Li X, Wang L, Yan S, Yang F, Xiang L, Zhu J, et al. Clinical characteristics of 25 death cases with COVID-19: a retrospective review of medical records in a single medical center, Wuhan, China. Int J Infect Dis. 2020

8. Mishra AK, Sahu KK, Lal A, Sargent J. Patterns of heart Injury in COVID - 19 and relation to outcome. J Med Virol. 2020 Apr 8. doi: 10.1002/jmv.25847.

9. Wang D, Hu B, Hu C, Zhu F, Liu X, Zhang J, et al. Clinical characteristics of 138 hospitalized patients with 2019 novel coronavirus-infected pneumonia in wuhan, China. JAMA. 2020; 323(11):1061-9.

10. Hu H, Ma F, Wei X, Fang Y. Coronavirus fulminant myocarditis saved with glucocorticoid and human immunoglobulin. Eur Heart J. 2020. https://doi. org/10.1093/eurheartj/ehaa190

11. Inciardi RM, Lupi L, Zaccone G, Italia L, Raffo M, Tomasoni D, et al. Cardiac involvement in a patient with coronavirus disease 2019 (COVID-19). JAMA Cardiol. 2020; 5(7):819-24.

12. Pan SF, Zhang HY, Li CS, Wang C. Cardiac arrest in severe acute respiratory syndrome: analysis of 15 cases. Zhonghua Jie He He Hu Xi Za Zhi. 2003; 26(10):602-5.

13. Oudit GY, Kassiri Z, Jiang C, Liu PP, Poutanen SM, Penninger JM, et al. SARScoronavirus modulation of myocardial ACE2 expression and inflammation in patients with SARS. Eur J Clin Invest. 2009; 39(7):618-25.
M, Icen YK; Redação do manuscrito: Koc M, Sumbul HE, Turunc T; Revisão crítica do manuscrito quanto ao conteúdo intelectual importante: Koc M, Karakoc E, Ozturk HA.

\section{Potencial Conflito de Interesses}

Declaro não haver conflito de interesses pertinentes.

\section{Fontes de Financiamento}

O presente estudo não teve fontes de financiamento externas.

\section{Vinculação Acadêmica}

Não há vinculação deste estudo a programas de pósgraduação.

14. Yang $C$, Jin Z. An acute respiratory infection runs into the most common noncommunicable epidemic - COVID-19 and cardiovascular diseases. JAMA Cardiol. 2020;5(7):743-4.

15. Ratliff NB, Estes ML, McMahon JT, Myles JL. Chloroquine-induced cardiomyopathy. Arch Pathol Lab Med. 1988; 112(6):578.

16. Seshadri MS, John L, Varkey K, Koshy TS. Ventricular tachycardia in a patient on dehydroemetine and chloroquine for amoebic liver abscess. Med J Aust. 1979; 1(9):406-7.

17. Roden DM, Harrington RA, Poppas A, Russo AM. Considerations for Drug Interactions on QTc in Exploratory COVID-19 (Coronavirus Disease 2019) Treatment. Circulation. 2020;141(24):e906-7.

18. Kongstad O, Xia Y, Liang Y, Hertervig E, Ljungström E, Olsson B, et al. Epicardial and endocardial dispersion of ventricular repolarization. A study of monophasic action potential mapping in healthy pigs. Scandinavian Cardiovascular Journal. 2005;39(6):342-7.

19. Porthan K, Viitasalo M, Toivonen L, Havulinna AS, Jula A, Tikkanen JT, et al. Predictive Value of Electrocardiographic T-Wave Morphology Parameters and T-Wave Peak to T-Wave End Interval for Sudden Cardiac Death in the General PopulationClinical Perspective. Circulation: Arrhythmia and Electrophysiology. 2013; 6(4):690-696.

20. Rautaharju PM, Surawicz B, Gettes LS, Bailey JJ, Childers R, Deal BJ, et al. AHA/ACCF/HRS recommendations for the standardization and interpretation of the electrocardiogram: part IV: the ST segment, T and $\mathrm{U}$ waves, and the QT interval. Endorsed by the International Society for Computerized Electrocardiology. J Am Coll Cardiol. 2009 Mar 17; 53(11):982-91.

21. Chua KC, Rusinaru C, Reinier K, Uy-Evanado A, Chugh H, Gunson K, et al. Tpeak-to-Tend interval corrected for heart rate: A more precise measure of increased sudden death risk? Heart rhythm. 2016; 13(11):2181-5.

22. Pieroni M, Smaldone C, Bellocci F. Myocarditis presenting with ventricular arrhythmias: role of electroanatomical mapping-guided endomyocardial biopsy in differential diagnosis.In: Cihakova D, editor. Myocarditis. InTech: University Johns Hopkins; 2011. p. 365-386.

23. Badorff C, Lee GH, Lamphear BJ, Martone ME, Campbell KP, Rhoads RE, et al. Enteroviral protease 2A cleaves dystrophin: evidence of cytoskeletal disruption in an acquired cardiomyopathy. Nat Med. 1999; 5(3): 320- 6 .

24. Ucar FM, Ozturk C, Yılmaztepe MA. Evaluation of Tp-e interval, Tp-e/QT ratio and Tp-e/QTc ratio in patients with acute myocarditis. BMC Cardiovasc Disord. 2019 Oct 22; 19(1): 232. 